\title{
The C609T (Pro187Ser) Null Polymorphism of the NQO1 Gene Contributes Significantly to Breast Cancer Susceptibility in North Indian Populations: a Case Control Study
}

\author{
Prasant Yadav $^{1}$, Rashid Mir ${ }^{2}$, Kajal Nandi ${ }^{1}$, Jamsheed Javid ${ }^{2}$, Mirza Masroor ${ }^{1}$, \\ Imtiyaz Ahmad', Mariyam Zuberi' ${ }^{1}$, RCM Kaza ${ }^{3}$, SK Jain ${ }^{3}$, Nita Khurana ${ }^{4}$, \\ Prakash Chandra Ray ${ }^{1}$, Alpana Saxena ${ }^{1 *}$
}

\begin{abstract}
Background: Worldwide, breast cancer is the most common cancer among women and is a leading cause of cancer death. In the present study, we investigated the NQO1 C609T genotypic and allelic distribution in north Indian breast cancer patients. Materials and Methods: The genotypic distribution of the NQ01 C609T polymorphism was assessed in 100 invasive ductal carcinoma (IDC) breast cancer patients and 100 healthy controls using allele specific PCR (AS-PCR). Results: A lower frequency of the CC genotype was found in breast cancer patients $(24 \%)$ than in the controls. On the other hand, TT genotype frequency was also found to be higher in female healthy controls $(32 \%)$ than the female breast cancer patients $(20 \%)$. The frequencies of all three genotypes CC, CT, TT in patients were $24 \%, 56 \%$ and $20 \%$ and in healthy controls $50 \%, 22 \%$ and $32 \%$ respectively. We did not find any significant correlation between the NQO1 C609T polymorphism and age group, grading, menopausal status and distant metastasis. A less significant association was found between the NQ01 C609T polymorphism and the stage of breast cancer $(X 2=5.931, P=0.05)$. Conclusions: The present study shows a strong association between NQO1 C609T polymorphism with the breast cancer risk in the north Indian breast cancer patients so that possible use as a risk factor should be further expel
\end{abstract}

Keywords: Breast cancer - NAD(P)H:quinoneoxidoreductase 1 polymorphism - invasive ductal carcinoma - India

Asian Pac J Cancer Prev, 17 (3), 1215-1219

\section{Introduction}

Breast cancer is the leading cause of cancer related female deaths all over the world accounting for $>1.6 \%$ of deaths and case fatality rates are highest in low-resource countries. In India, Breast cancer is the most frequently occurring cancers in females in comparison with other cancers. In India the average age of the high risk group for breast cancer is 43-46 years unlike in the west where women aged 53-57 years are more prone to breast cancer.

NADPH: Quinone oxidoreductase 1(NQO1) gene, originally called DT-diaphorase is located on the 16q22.1. NQO1 gene codes a 274 amino acids (30868 Da) containing cytosolic NQO1 enzyme which catalyzes the two-electron reduction of quinone compounds and prevents the generation of semi-quinone free radicals and reactive oxygen species, thus protecting cells from oxidative damage. Additionally, NQO1 catalyzes the reductive activation of quinoid chemotherapeutic agents and of environmental carcinogens such as nitrosamines, heterocyclic amines and cigarette smoke condensate (Rauth et al., 1997). There is evidence that oxidative stress, resulting from either an excess of reactive oxygen species (ROS) or a deficiency in antioxidant capabilities, may play a role in the etiology of breast cancer (Nowell et al., 2004, Ambrosone et al., 2000).

NQO1 was found to play an important role in apoptosis and oxidative stress, and the effects of C609T polymorphism were thoroughly investigated in several types of cancers (Lewis et al., 2001, Menzel et al., 2004, Hamajima et al., 2002, Niwa et al., 2005, di Martino et al., 2007). NAD(P)H:quinone oxidoreductase 1 (NQO1) may be particularly relevant for breast carcinogenesis because of its role in reduction of endogenous catechol estrogens generated in the metabolism of estrogen. By catalyzing the obligatory two-electron reduction of catechol estrogens and other quinones, the reactive semiquinone intermediate that drives the Fenton reaction is bypassed, and superoxide 


\section{Prasant Yadav et al}

mediated release of iron from ferritin stores is prevented (Wyllie et al., 1997). The gene coding for NQO1 is subject to polymorphism (C609T) with a $\mathrm{C} / \mathrm{T}$ base change at position 609 of the human NQO1 cDNA, leading to a change in the amino acid sequence of the protein (P187S).

The C609T polymorphism (rs1800566) in NQO1 (Traver et al., 1997) leads to a proline to serine substitution in the NQO1 protein that results in loss of virtually all enzyme activity due to rapid degradation of the variant enzyme (Ross et al., 1996, Siegel et al., 1999). This major polymorphism involves a single $\mathrm{C}$ to $\mathrm{T}$ substitution at nucleotide 609 of exon 6 of the NQO1 cDNA that causes a Pro187Ser amino acid change thereby leading to three phenotypes, i.e. wild-type phenotype with complete enzyme activity, heterozygous phenotype with $\sim 3-$ fold decreased activity and homozygous mutant (null) phenotype with a complete lack of enzyme activity (Kuehl, B.L.et al 1995, Moran, J.L. et al 1999). Decreased NQO1 enzyme activity is caused by enhanced polyubiquination and proteasomal degradation of the mutant protein (Siegel, D. et al. 2001). In the current study, we investigated the NQO1 C609T genotypic and allelic distribution and its role in breast cancer patients from north Indian population.

\section{Materials and Methods}

During 2010 to 2012, from 100 female healthy controls and 100 female patients of confirmed Invasive Ductal Carcinoma (IDC) breast cancer in Department of Surgery, Maulana Azad Medical College, New Delhi assessed on the basis of clinic-pathological findings, a 5 $\mathrm{ml}$ sample of peripheral blood was collected from each patient and healthy control. Patients with any previous history of cancer were excluded from this study. This study was approved by the Institutional Ethical Committee of Maulana Azad Medical College, New Delhi. The clinical characteristics of the patients and healthy controls are given in Table 1.

Whole genomic DNA extraction was done from whole blood by DNA extraction kit (Geneaid, India) according to manufacturer's protocol. NQO1 C609T polymorphism was evaluated by the AS-PCR using previously described primers (Hamajima N, 2002). The total volume of the PCR reaction was $25 \mu l$ and the amplification was done using the conditions; 100ng genomic DNA, $0.25 \mu 1$ of $25 \mathrm{pmol} /$ $\mu 1$ each primers, $2.5 \mu 110 \mathrm{mM}$ dNTPs, $1.5 \mu 1$ of $20 \mathrm{mM}$ $\mathrm{MgCl} 2,0.3 \mu 1$ of $5 \mathrm{U} / \mu 1 \mathrm{Taq}$ polymerase with $2.5 \mu 1$ of 10X Taq Buffer (Fermantas). The PCR program for NQO1 C609T polymorphism was initial denaturation at 95 oc for 10 minutes followed by 40 cycles $95^{\circ} \mathrm{c}$ for $45 \mathrm{sec}, 55$ oc for $45 \mathrm{sec}$ followed by the final extension at $72 \mathrm{oc}$ for 10 minutes. Agarose gel electrophoresis was performed on the $2 \%$ agarose gel for analysis of amplicon product.

\section{Statistical analysis}

Statistical analysis was done using SPSS16 and MedCalc software. The estimated risk for both alleles was analyzed using Odd ratios. Standard chi-square test was used to calculate the p-value and the p-value less than 0.05 was taken as significant.

\section{Results}

Patients' clinicopathological characteristics are shown in table 1 . Of 100 consecutive breast cancer patients blood samples collected $49(49 \%)$ patients were below or equal to 45 years age and $51(51 \%)$ were above 45 . Besides $44(44 \%)$ patients were premenopausal status and 56(56\%) patients were postmenopausal. Of breast cancer cases 44(44\%) were in early (I \& II) stages and 56(56\%) samples were in advanced stages (III \& IV). Histological grading of the patients' tumor showed that $17(17 \%), 52(52 \%)$ and $31(31 \%)$ were in grade I, II and III respectively. Lymph node status of patients showed that 59(59\%) were lymph node positive and $7(7 \%)$ patients had distant metastasis of total samples. Of the total samples 54(54\%) samples were Her2/neu positive.

The genotypic distribution of NQ01 C609T polymorphism in breast cancer patients with respect to female healthy controls showed a significant correlation $\left(X^{2}=26.6, d f=2, p<0.0001\right)$ (Table 2). A lower frequency

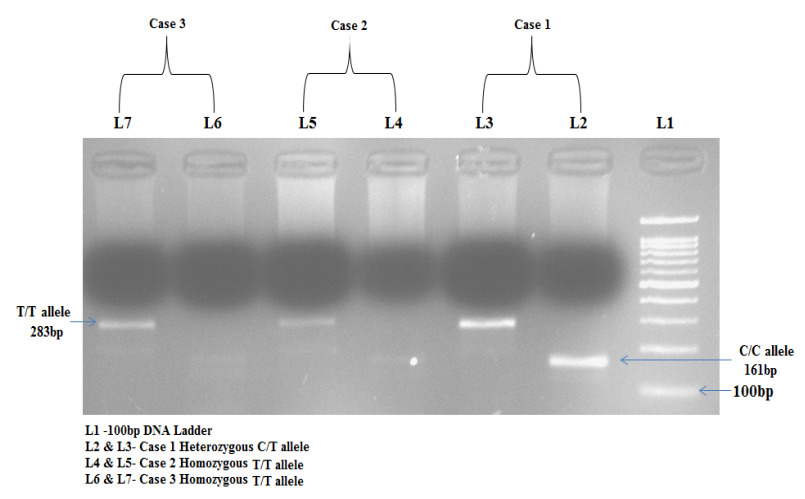

Figure 1. Agarose Gel Electrophoresis of AS-PCR of NQ01 C609T Alleles

Table 1. Clinico-pathological Characteristics of Breast Cancer Patients

\begin{tabular}{lcc}
\hline $\begin{array}{l}\text { Parameters } \\
\text { Patients }\end{array}$ & $\begin{array}{c}\text { Patients (\%) } \\
100(100)\end{array}$ & $\begin{array}{c}\text { Healthy Controls (\%) } \\
100(100)\end{array}$ \\
\hline $\begin{array}{l}\text { Age Group } \\
\text { Age }>45\end{array}$ & $49(49)$ & \\
Age $<45$ & $51(51)$ & \\
Menopause & & \\
$\quad$ Pre & $44(44)$ \\
$\quad$ Post & $56(56)$ \\
Stage & \\
$\quad$ Early(I \& Ii) & $44(44)$ \\
$\quad$ Advanced (Ii \& Iv) & $56(56)$ \\
Grading & \\
I & $17(17)$ \\
Ii & $52(52)$ \\
Iii & $31(31)$ \\
Lymph Nodes & \\
Positive & $59(59)$ \\
Negative & $41(41)$ \\
Her2/Neu & \\
Positive & $54(54)$ \\
Negative & $46(46)$ \\
Distant Metastasis & \\
Positive & $7(7)$ \\
Negative & $93(93)$ \\
\hline & \\
\hline
\end{tabular}


Table 2. NQO1 (C609T) Polymorphism with Different Clinical Features of Breast Cancer Patients

\begin{tabular}{|c|c|c|c|c|c|c|}
\hline Parameters & Total Samples & $\mathrm{Cc}$ & $\mathrm{Tc}$ & $\mathrm{Tt}$ & $\mathrm{X}^{2}$ & P-Val \\
\hline Patients & 100 & $24(24 \%)$ & $56(56 \%)$ & $20(20 \%)$ & 26.6 & $<0.0001$ \\
\hline Controls & 100 & $50(50 \%)$ & $22(22 \%)$ & $32(32 \%)$ & & \\
\hline \multicolumn{7}{|l|}{ Age Group } \\
\hline Below Or $=45$ & 49 & $12(24.5 \%)$ & $26(53.1 \%)$ & $11(22.4 \%)$ & \multirow[t]{2}{*}{0.44} & \multirow[t]{2}{*}{$0.80(\mathrm{Ns})$} \\
\hline Above 45 & 51 & $12(23.5 \%)$ & $30(58.9 \%)$ & $9(17.6 \%)$ & & \\
\hline \multicolumn{7}{|l|}{ Menopause } \\
\hline Pre & 44 & $12(27.3 \%)$ & $23(52.3 \%)$ & $9(20.4 \%)$ & \multirow[t]{2}{*}{0.55} & \multirow[t]{2}{*}{$0.75(\mathrm{Ns})$} \\
\hline Post & 56 & $12(21.5 \%)$ & $33(58.9 \%)$ & $11(19.6 \%)$ & & \\
\hline \multicolumn{7}{|l|}{ Stage } \\
\hline Early(I \& Ii) & 44 & $15(34.1 \%)$ & $19(43.2 \%)$ & $10(22.7 \%)$ & \multirow[t]{2}{*}{5.931} & \multirow[t]{2}{*}{0.05} \\
\hline Advanced (Iii \& Iv) & 56 & $9(16.1 \%)$ & $37(66.1 \%)$ & $10(17.8 \%)$ & & \\
\hline \multicolumn{7}{|l|}{ Grading } \\
\hline I & 17 & $4(23.5 \%)$ & $5(29.4 \%)$ & $8(47.1 \%)$ & & \multirow[t]{3}{*}{ Ns } \\
\hline $\mathrm{Ii}$ & 52 & $14(26.9 \%)$ & $28(53.9 \%)$ & $10(19.2 \%)$ & & \\
\hline Iii & 31 & $6(19.6 \%)$ & $23(74.2 \%)$ & $2(6.5 \%)$ & & \\
\hline \multicolumn{7}{|l|}{ Lymph Nodes } \\
\hline Positive & 59 & $7(11.9 \%)$ & $42(71.2 \%)$ & $10(16.9 \%)$ & \multirow[t]{2}{*}{15.42} & \multirow[t]{2}{*}{0.0004} \\
\hline Negative & 41 & $17(41.5 \%)$ & $14(34.1 \%)$ & $10(24.4 \%)$ & & \\
\hline \multicolumn{7}{|l|}{ Her2/Neu Status } \\
\hline Positive & 54 & $9(16.6 \%)$ & $29(54.8 \%)$ & $16(29.6 \%)$ & \multirow[t]{2}{*}{8.18} & \multirow[t]{2}{*}{0.01} \\
\hline Negative & 46 & $15(32.6 \%)$ & $27(58.7 \%)$ & $4(8.7 \%)$ & & \\
\hline \multicolumn{7}{|l|}{ Distant Metastasis } \\
\hline Positive & 7 & $0(0 \%)$ & $6(85.7 \%)$ & $1(14.3 \%)$ & & \multirow[t]{2}{*}{$0.23(\mathrm{Ns})$} \\
\hline Negative & 93 & $24(0 \%)$ & $50(0 \%)$ & $19(0 \%)$ & & \\
\hline
\end{tabular}

Table 3. Comparison of Tumor Grading with Different Genotypes of NQO1 C609T Polymorphism in Breast Cancer Patients

\begin{tabular}{|c|c|c|c|c|c|}
\hline \multicolumn{6}{|c|}{ Grading Comparison } \\
\hline Grade vs Grade & TOTAL PATIENTS & $\mathrm{CC}$ & $\mathrm{CT}$ & $\mathrm{TT}$ & P-value \\
\hline \multirow[t]{2}{*}{ I vsII } & 17 & $4(23.5 \%)$ & $5(29.4 \%)$ & $8(47.1 \%)$ & $0.09(\mathrm{NS})$ \\
\hline & 52 & $14(26.9 \%)$ & $28(53.9 \%)$ & $10(19.2 \%)$ & \\
\hline \multirow[t]{2}{*}{ I vs III } & 17 & $4(23.5 \%)$ & $5(29.4 \%)$ & $8(47.1 \%)$ & 0.001 \\
\hline & 31 & $6(19.6 \%)$ & $23(74.2 \%)$ & $2(6.5 \%)$ & \\
\hline \multirow{2}{*}{ II vs III } & 52 & $14(26.9 \%)$ & $28(53.9 \%)$ & $10(19.2 \%)$ & $0.13(\mathrm{NS})$ \\
\hline & 31 & $6(19.6 \%)$ & $23(74.2 \%)$ & $2(6.5 \%)$ & \\
\hline
\end{tabular}

of $\mathrm{CC}$ genotype was found in breast cancer Patients $(24 \%)$ than the female healthy controls $(50 \%)$. On the other hand, TT genotype frequency was also observed to be lower in female breast cancer patients $(20 \%)$ than the female healthy controls $(32 \%)$. Frequencies of all three genotypes CC, CT, TT in patients are $24 \%, 56 \%$ and $20 \%$ and in healthy controls were $50 \%, 22 \%$ and $32 \%$ respectively. We were not able find any significant association between the NQO1 C609T polymorphism and age, histological grading, menopausal status and presence/ absence of distant metastasis.

A low significant association was found between the NQ01 C609T polymorphism with the early/advanced stage of cancer $\left(\mathrm{X}^{2}=5.931, \mathrm{p}=0.05\right)$ (Table 2). Early stage group included Ductal Carcinoma in Situ (DICS) patients in stage I and II of breast cancer. On the other hand, advanced stage group included breast cancer patients in stage III and IV.

During the assessment of correlation between NQ01 C609T Polymorphism with lymph node status of breast cancer patients we observed a highly significant correlation $\left(\mathrm{X}^{2}=15.42, \mathrm{P}=0.0004\right)$ (Table 2$)$.
We have found a significant association between NQ01 C609T polymorphism and HER2/neu positive status of breast cancer patients $\left(\mathrm{X}^{2}=8.18, \mathrm{P}=0.01\right)$ (Table: 2$)$. During the investigation of association of NQ01 C609T polymorphism with histological tumor grading of breast cancer patients, we observed a significant result during comparison of tumor grade I vs III ( $\mathrm{p}=0.001$ ). However, we were not able to find any significant association between tumor grades I vs II ( $\mathrm{p}=0.09)$ or grade II vs III $(\mathrm{p}=0.13)$ (Table 3).

In the investigation of NQO1 C609T polymorphism with breast cancer risk we have found a highly significant association. We observed significant results in analysis of both allelic as well as genotypic distribution (Table 4). During the comparison of CC homozygous genotype with the TT homozygous genotype we were not able to find any significant results for OR 1.30(0.73-1.91) RR 1.18 (0.73-1.91) $(\mathrm{p}=0.48)$. In comparison of CC homozygous genotype with the CT heterozygous genotype we found a highly significant association with the OR 5.30 (2.6510.6) RR 2.21 (1.54-3.56) ( $\mathrm{P} \leq 0.0001)$. During the allelic comparison, the $\mathrm{C}$ allele was compared with the $\mathrm{T}$ allele 
Table 4. Risk of Breast Cancer Associated with the NQO1 C609T Polymorphism

\begin{tabular}{|c|c|c|c|c|c|c|c|}
\hline & \multicolumn{2}{|c|}{$\begin{array}{c}\text { Breast cancer } \\
\text { patients }(\mathrm{N}=100)\end{array}$} & \multicolumn{2}{|c|}{$\begin{array}{l}\text { Healthy controls } \\
\qquad(\mathrm{N}=100)\end{array}$} & \multirow[t]{2}{*}{ OR $(95 \% \mathrm{CI})$} & \multirow[t]{2}{*}{ Risk Ratio(RR) } & \multirow[t]{2}{*}{ P-Val } \\
\hline & No. & $\%$ & No. & $\%$ & & & \\
\hline Pro/Pro (CC) & 24 & $-24 \%$ & 50 & $-50 \%$ & 1(ref.) & 1(ref.) & \\
\hline Pro/Ser (CT) & 56 & $-56 \%$ & 22 & $-22 \%$ & $5.30(2.65-10.6)$ & $2.21(1.54-3.56)$ & $<0.0001$ \\
\hline Ser/Ser (TT) & 20 & $-20 \%$ & 32 & $-32 \%$ & $1.30(0.62-2.73)$ & $1.18(0.73-1.91)$ & $0.48(\mathrm{NS})$ \\
\hline Pro/Ser + Ser/Ser $(C C+$ TT $)$ & 76 & & 54 & & $2.93(1.61-5.33)$ & $1.80(1.25-2.58)$ & 0.0004 \\
\hline Pro & 104 & & 122 & & 1(ref.) & 1(ref.) & \\
\hline Ser & 96 & & 86 & & $1.30(0.88-1.93)$ & $1.14(0.94-1.39)$ & $0.17(\mathrm{NS})$ \\
\hline
\end{tabular}

Table 5. Allelic Frequencies of NQO1 C609T Polymorphism in Breast Cancer Patients

\begin{tabular}{|c|c|c|c|c|c|c|}
\hline & \multicolumn{6}{|c|}{ Allelic Frequencies } \\
\hline & $\begin{array}{c}\text { Total } \\
\text { Samples }\end{array}$ & $\mathrm{Cc}$ & $\mathrm{Tc}$ & $\mathrm{Tt}$ & $\begin{array}{c}\text { C Allele } \\
\text { Frequency }\end{array}$ & $\begin{array}{r}\text { T Allele } \\
\text { Frequency }\end{array}$ \\
\hline Patients & 100 & $24(24 \%)$ & $56(56 \%)$ & $20(20 \%)$ & 0.52 & 0.48 \\
\hline Controls & 100 & $50(50 \%)$ & $22(22 \%)$ & $32(32 \%)$ & 0.59 & 0.41 \\
\hline$X^{2}=26.6$ & P-Val $\leq 0.0001$ & Df $=2$ & & & & \\
\hline
\end{tabular}

and we observed an odd ratio (OR) $1.30(0.88-1.93)$ and risk ratio $(\mathrm{RR}) 1.14(0.94-1.39)(\mathrm{P}=0.17)$.

\section{Discussion}

$\mathrm{NAD}(\mathrm{P}) \mathrm{H}$ : quinone oxireductase (NQO1) is an enzyme that is involved in metabolising numerous endogenous quinones. Exchange of Proline at position 187 by Serine leads to a non-functional enzyme (Nebert DW et al. 2002). Individuals homozygous for the 187 serine allele have a high risk for aplastic anaemia and leukaemia. Present study showed a strongly positive association between NQO1 C609T polymorphism with breast cancer risk in north Indian breast cancer patients. We have shown a significant association between NQO1 C609T polymorphism and different patients characteristics (stage, lymph node status, Her2/neu status). Another study published by V Singh et al (2011) on north Indian breast cancer patients concluded that NQO1 C609T polymorphism does not play a significant role in breast cancer susceptibility in north Indian women. Fagerholm et al (2008) suggested that NQO1 was a strong prognostic and predictive factor in breast cancer patients receiving anthracyclines, and they also proposed that NQO1 influences the outcome of epirubicin treatment through at least three mechanisms: the TP53 and TNF-NF-kB pathways and direct detoxification of reactive oxygen species. H-J Menzel et al (2004) studied in breast cancer patients from two different populations and found that the $187 \mathrm{~S}$ allele was found significantly more frequently in breast cancer patients than in controls. However our results suggest that the heterozygous genotype has a higher risk of breast cancer than homozygous $187 \mathrm{~S}$ genotype. Our results also suggest that NQO1 C609T polymorphism may be significantly associated with the advancement of breast cancer stage. J Sarmanova et al (2004) has suggested an influence of genetic polymorphisms of xenobiotic-metabolizing enzymes, particularly NQO1, on the susceptibility to breast cancer, possibly by change of the ratio of activation/ detoxification of procarcinogens or by linkage to another cancer-causative gene(s). Fowke et al (2004) suggested that the use of oral contraceptives was associated with a lower breast cancer risk among women with the T allele of NQO1.

The C609T NQO1 polymorphism is one of very few known and common single nucleotide polymorphisms that completely eliminate enzymatic activity, and its biological impact in vivo is undoubted The NQO1 enzyme has been increasingly attracting attention in cancer and more roles unrelated to its classical metabolic functions are being constantly discovered. The various functions NQO1 performs are - NQO1 enzyme has the classical role of catalysing the obligatory two electron reduction of a broad range of exogenous and endogenous quinones to their respective hydroquinones, preventing the one electron reduction of these compounds into semiquinones and a variety of reactive oxygen species. NQO1 was found to have an additional antioxidant effect by directly and independently scavenging superoxides, a function that is yet to be established. NQO1 enzyme was recently found to act as a stabilizer for several tumor suppressor proteins (p53, p73, and p33), and this stabilization was shown to be especially prominent under conditions of oxidative stress. As the C609T polymorphism principally affects the susceptibility of the enzyme to degradation in vivo, it affects all aspects of NQO1 function indistinguishably. The largest role of NQO1 in cancer susceptibility is attributable to the interaction of the enzyme with environmental exposure. The high minor allelic frequency of the C609T polymorphism of this 'environmental' enzyme in the Asian population (43\%) compared with the Caucasian population $(20 \%)$ suggests that the environmental exposure is widely different among the two populations. The variation in allele frequency of this polymorphism therefore highlights major environmental differences among the two populations and may in turn partly explain why a significant association was found in Caucasians but not in Asians. Another possible explanation is the widely different genetic structure between the two populations, as the overall effects of NQO1 C609T polymorphism 
on cancer susceptibility might be overshadowed or compensated by variants in other metabolic genes. In conclusion, present study demonstrates that the NQO1 C609T polymorphism may increase the risk of breast cancer in north Indian population.

In conclusion, The present study suggests the NQO1 C609T polymorphism as an important genetic factor in the overall risk for developing breast cancer, especially in Indian populations and predicts that oxidative stressmodifying enzymes would be a promising area of further investigation for prognostic markers of breast cancer. However, more association studies are needed to support this finding in various ethnic groups.

\section{Acknowledgements}

The authors thank the participants from Maulana Azad Medical College, New Delhi, for their cooperation and participation.

\section{References}

Ambrosone CB (2000). Oxidants and antioxidants in breast cancer. Antioxid Redox Signal, 2, 903-17.

di Martino E1, Hardie LJ, Wild CP, et al (2007). "The NAD(P) $\mathrm{H}$ :quinone oxidoreductase I C609T polymorphism modifies the risk of barrett esophagus and esophageal adenocarcinoma." Genetics Med, 9, 341-7.

Fagerholm R, Hofstetter B, Tommiska J, et al (2008). NAD(P) $\mathrm{H}$ :quinone oxidoreductase $1 \mathrm{NQO} 1 * 2$ genotype $(\mathrm{P} 187 \mathrm{~S})$ is a strong prognostic and predictive factor in breast cancer. Nat Genet, 40, 844-53.

Hamajima N, Matsuo K, et al (2002). NAD(P)H: quinone oxidoreductase 1 (NQO1) C609T polymorphism and the risk of eight cancers for Japanese. Int J Clin Oncol, 7, 103-8.

Jana Sarmanova, Simona Su sova, Ivan Gut, et al (2004). Breast cancer: role of polymorphisms in biotransformation enzymes. Eur J Human Genetics, 12, 848-54.

Jay H. Fowke, Xiao-Ou Shu, Qi Dai, et al (2004). Genetic Polymorphisms Modification by NAD(P)H:Quinone oxoreductase (NQO1) oral contraceptive use and breast cancer risk. cancer epidemiol biomarkers prev, 13, 1308-15.

Kuehl BL, Paterson JW, Peacock JW, et al (1995). Presence of a heterozygous substitution and its relationship to DTdiaphorase activity. Br J Cancer, 72, 555-561.

Lewis SJ, NM Cherry (2001). Polymorphisms in the NAD(P)H: quinone oxidoreductase gene and small cell lung cancer risk in a UK population. Lung Cancer, 34, 177-183.

Menzel HJ, J Sarmanova, et al (2004). Association of NQO1 polymorphism with spontaneous breast cancer in two independentpopulations. Br J Cancer, 90, 1989-94.

Moran JL, Siegel D, Ross D (1999). A potential mechanism underlying the increased susceptibility of individuals with a polymorphism in $\mathrm{NAD}(\mathrm{P}) \mathrm{H}$ quinone oxidoreductase 1 (NQO1) to benzene toxicity. Proc Natl Acad Sci USA, 96, $8150-5$

Menzel HJ, J Sarmanova (2004). Association of NQO1 polymorphism with spontaneous breast cancer in two independent populations. Br J Cancer, 90, 1989-94.

Nowell SA, Ahn J, Ambrosone CB, et al (2004). Gene-nutrient interactions in cancer etiology. Nutr Rev, 62, 427-38.

Niwa Y,K Hirose (2005). Association of the NAD(P)H: quinone oxidoreductase $\mathrm{C} 609 \mathrm{~T}$ polymorphism and the risk of cervical cancer in Japanese subjects. Gynecologic Oncol 96, 423-9.

Nebert DW, Roe AL, Vandale SE, Bingham E, Oakley GG (2002)
$\mathrm{NAD}(\mathrm{P}) \mathrm{H}$ :quinone oxidoreductase (NQO1) polymorphism, exposure to benzene, and predisposition to disease: a huge review. Genet Med, 4, 62-70

Rauth AM, Goldberg Z, Misra V (1997) DT-diaphorase: possible roles in cancer. chemotherapy and carcinogenesis. Oncol Res, 9, 339-49.

Ross D, Traver RD, Siegel D, et al (1996). A polymorphism in $\mathrm{NAD}(\mathrm{P}) \mathrm{H}$ : quinone oxidoreductase (NQO1): relationship of a homozygous mutation at position 609 of the NQO1 cDNA to NQO1 activity. Br J Cancer, 74, 995-6.

Siegel D, McGuinness SM, Winski SL, Ross D (1999). Genotypephenotype relationships in studies of a polymorphism in $\mathrm{NAD}(\mathrm{P}) \mathrm{H}$ :quinone oxidoreductase 1. Pharmacogenetics 9, 113-21.

Siegel D, Anwar A,Winski SL, Kepa JK, Dowd KL (2001). Rapid polyubiquination and proteasomal degradation of a mutant form of $\mathrm{NAD}(\mathrm{P}) \mathrm{H}$ : quinone oxidoreductase $1 . \mathrm{Mol}$ Pharmacol, 59, 263-8.

Singh V, Upadhyay G, Rastogi N, Singh K, Singh MP (2011) Polymorphism of xenobiotic-metabolizing genes and breast cancer susceptibility in North Indian women. Genet Test Mol Biomarkers, 15, 343-349.

Traver RD, Siegel D, Beall HD, et al (1997) Characterization of a polymorphism in $\mathrm{NAD}(\mathrm{P}) \mathrm{H}$ : quinone oxidoreductase (DT-diaphorase). Br J Cancer, 75, 69-75.

Wyllie S, Liehr JG (1997) Release of iron from ferritin storage by redox cyclingof stilbene and steroid estrogen metabolites: a mechanism of induction of free radical damage by estrogen. Arch Biochem Biophys, 346, 180-6. 